Research Article

\title{
Fluid-Induced Nonlinear Vibration of a Cantilevered Microtube with Symmetric Motion Constraints
}

\author{
Yong Guo \\ School of Civil Engineering, Guizhou Institute of Technology, Guiyang, China \\ Correspondence should be addressed to Yong Guo; gy-gates@163.com
}

Received 26 March 2020; Revised 22 June 2020; Accepted 9 July 2020; Published 12 August 2020

Academic Editor: Francesco Franco

Copyright (๑) 2020 Yong Guo. This is an open access article distributed under the Creative Commons Attribution License, which permits unrestricted use, distribution, and reproduction in any medium, provided the original work is properly cited.

\begin{abstract}
This paper investigates the dynamic behavior of a cantilevered microtube conveying fluid, undergoing large motions and subjected to motion-limiting constraints. Based on the modified couple stress theory and the von Kármán relationship, the strain energy of the microtube can be deduced and then the governing equation of motion is derived by using the Hamilton principle. The Galerkin method is applied to produce a set of ordinary differential equations. The effect of the internal material length scale parameter on the critical flow velocity is investigated. By using the projection method, the Hopf bifurcation is demonstrated. The results show that size effect on the vibration properties is significant.
\end{abstract}

\section{Introduction}

The dynamics of microtube conveying fluid is of considerable interest in engineering and has been studied widely over the past few decades [1-9]. Holmes [10] proved that pipes supported at both ends cannot flutter. The Hopf bifurcation of a cantilevered tube carrying fluid was investigated theoretically by Bajaj et al. [11], revealing that the type of the Hopf bifurcation being supercritical or subcritical depends on the mass ratio and friction factor in a complicated manner. The fluid-conveying tube was modified to include motion-limiting restraints and a full nonlinear analysis was given by Paidoussis and Semler [12]. In [13] by Jin, a linear spring support was attached to the system of Paidoussis et al. [8], and the critical velocity and the chaotic motions of this system were investigated. A variant of the basic system, that is, a standing pipe conveying fluid was studied by Wang and $\mathrm{Ni}$ [14], and the dynamics of this variant was also compared with that of a hinging system developed by Jin [13]. By applying the multidimensional Lindstedt-Poincaré method, a clampedclamped fluid-conveying pipe under external periodic excitation was studied by Liang and Wen [15]. Based on the Galerkin truncation of order 8 and the numerical analysis, the dynamic behaviors of fluid-conveying pipes for three types of supports with lateral motion were discussed by Li et al. [16].
Zhang et al. [17] investigated the nonlinear forced vibration of fluid-conveying pipes in the supercritical regime, that is, vibration about a curved equilibrium.

Due to recent technological development in science and technology, the characteristic size of pipes becomes smaller and smaller. In [18] by Rinaldi et al., the inside diameter of the circular microtube ranges from 1 to $100 \mu \mathrm{m}$. However, regarding its application in microelectronic-mechanical-systems and engineering microfluidic devices, as an example, microtube conveying fluid can be applied to a class of microresonators $[19,20]$ and be considered to deliver drugs in targeted cancer therapy [21]. In recent years, there has been a great deal of interest in the static and dynamic behavior of pipe (or beam) at micro- and nanoscales [22-27]. Since the size-dependent behavior of microscale structure has been observed experimentally [28-30], we cannot directly extend the analysis of macroscale structures to that of microscale structure. Thus, nonclassical elasticity theories have to been introduced in order to incorporate the size dependence. Recently, a modified couple stress theory capable of capturing the size effect was developed by Yang et al. [31], in which the couple stress tensor is symmetrical and only one internal material length scale parameter is involved. Park and Gao [25] applied the theory to study the static mechanical properties of a Euler-Bernoulli beam. Asghari et al. [27] presented a nonlinear size-dependent Timoshenko 
beam model based on the modified couple stress theory. In their work, the geometric nonlinearity represented by the von Kármán relationship was adopted to derive the governing equation of motion. For fluid-conveying pipe with microscale, a new theoretical model was developed by Wang [22] for the linear vibration analysis, in which the Euler-Bernoulli model assumption and the modified couple stress theory were employed. In another paper by Xia and Wang [26], the sizedependent vibration analysis of microtube was extended to the Timoshenko model. Yang et al. [23] investigated the microfluid-induced nonlinear free vibration of microtube with both immovable ends by using the modified couple stress theory. In their paper, the geometry nonlinearity arising from the midplane stretching was taken into account, and the static postbuckling problem was discussed. Based on the nonlocal strain gradient theory and the Rayleigh beam theory, the wave dispersion in viscoelastic lipid nanotubes conveying protein solution, such as the relationship between wave frequency and wave number and the effect of the wave number and damping coefficient on the stability of tubes, was investigated by Cao and Wang [32]. In contrast to the past literature using the beam theory to model the pipe structures, Wang et al. [33] as well as $\mathrm{Li}$ and Wang [34] investigated the vibration behavior of fluidconveying nanotubes using the shell model.

Perhaps the first study of the dynamics of cantilevered micropipes conveying fluid was contributed by Hosseini and Bahaadini [35], who derived the linear governing equation of motion based on the modified strain gradient theory and then carried out an analysis of eigenvalues with a parametric study in order to examine the effect of the length scale parameter. In another paper by Bahaadini and Hosseini [36], the effect of the fluid slip condition on the free vibration and the flutter instability of viscoelastic cantilevered carbon nanotubes (CNTs) conveying fluid was investigated. The material property of the CNT was simulated by the Kelvin-Voigt viscoelastic constitutive relationship. The equations derived by Hosseini and Bahaadini are linear. Hu et al. [37] developed a nonlinear two-dimensional model for cantilevered fluidconveying micropipes and explored the possible size-dependent nonlinear responses based on the modified couple stress theory. Guo et al. [38] applied the center manifold theory, normal form method and symmetry to reduce rigorously the equations of motion to a two-degree-of-freedom dynamic system and calculate the corresponding coefficients. Furthermore, the averaging method was employed to investigate the reduced equations for analyzing two types of periodic motions, that is, the planar periodic motions and the spatial periodic motions, along with their stabilities.

However, to the best of our knowledge, no nonlinear vibration model of cantilevered microtube, undergoing large motions and subjected to motion-limiting constraints, is available to date. In this paper, we present a nonlinear model to account for the size effect on the dynamics of cantilevered microtube. The system is modeled as Euler-Bernoulli microtube. The geometry nonlinearity is taken into account by introducing the von Kármán relationship. By the use of modified couple stress theory, the strain energy of the microtube can be calculated. The kinetic expression of the system is available. The Hamilton principle is utilized to derive the governing equation of motion. The application of the Galerkin expansion and modal truncation techniques leads to a set of ordinary differential equations. With the projection method, the Hopf bifurcation is analyzed technically. Finally, the size effect on the dynamics of the system is discussed.

\section{Derivation of the Governing Equation of Motion}

The system subjected to motion constraints consists of a microtube of length $L$, cross-sectional area $A_{p}$, flexural rigidity $\mathrm{EI}$, and mass per unit length $m$, conveying an incompressible fluid of mass $M$ per unit length, flowing axially with velocity $V$ not varying with time. The cross section of the microtube is assumed to be symmetric, either circular or rectangular. Using the rectangular Cartesian coordinate system $(x, y, z)$, where the $x$-axis is coincident with the centroidal axis of the undeformed tube, the $y$-axis is the neutral axis and the $z$-axis is the symmetric axis as shown in Figure 1.

For a slender microtube with a large aspect ratio, the Euler-Bernoulli beam theory can be employed to obtain the displacement components of a point $(s, y, z)$ on a pipe cross section, with $s$ being the arc length along the centroidal axis, usually written as [25]

$$
\begin{aligned}
& u_{x}=u-z \psi \\
& u_{y}=0 \\
& u_{z}=w
\end{aligned}
$$

In the above equations, $u$ and $w$ represent the displacements of the centerline of the pipe with the coordinate $(s, 0,0)$ in the $x$-direction and the $z$-direction, respectively. $\psi$ is the rotation angle of the cross section with respect to the $z$-axis. For the case of a cantilevered tube without axial force, it is reasonable to regard the centerline to be inextensible, which leads to [39]

$$
\left(1+\frac{\partial u}{\partial s}\right)^{2}+\left(\frac{\partial w}{\partial s}\right)^{2}=1
$$

Based on the Euler-Bernoulli beam theory, the cross sections of the pipe remain perpendicular to the deformed centroidal line. Hence, we find that [38]

$$
\sin \psi=\frac{\partial w}{\partial s} \text {. }
$$

Before calculating the strain energy, the utilization of the modified couple stress theory will be reviewed first. For more details on this topic, the interested reader may refer to $[31,40]$. According to the modified couple theory, the strain energy density is a function of both the strain tensor and the curvature tensor. Furthermore, the strain energy $U$ in a deformed isotropic linear elastic material occupying region $\Omega$ can be written as $[23,27]$

$$
U=\frac{1}{2} \int_{\Omega}\left(\sigma_{i j} \varepsilon_{i j}+m_{i j} \chi_{i j}\right) \mathrm{d} v
$$

where the stress tensor $\sigma_{i j}$, the strain tensor $\varepsilon_{i j}$, the deviatoric part of the couple stress tensor $m_{i j}$, and the symmetric curvature tensor $\chi_{i j}$ are given by 

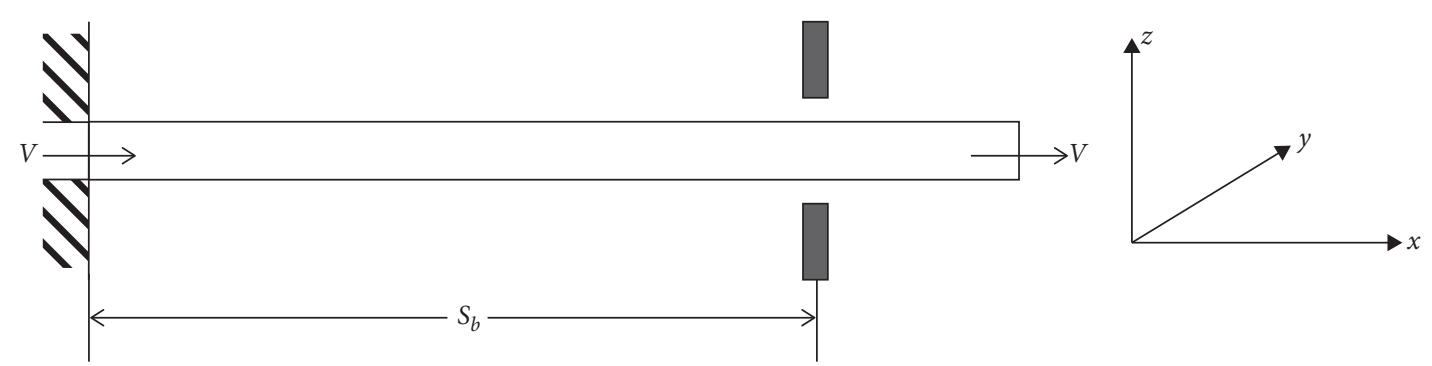

(a)

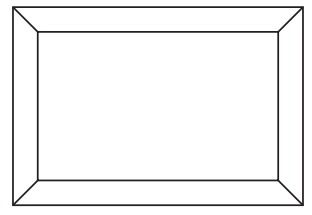

(c)

(b)

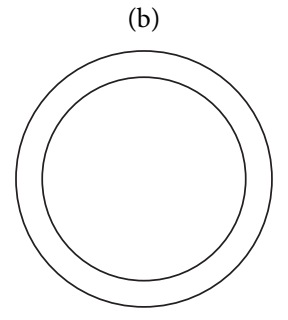

(d)

FIGURE 1: Schematic of a fluid-conveying microtube with one end free and subjected to motion constraints.

$$
\begin{aligned}
& \boldsymbol{\sigma}=\lambda \operatorname{tr}(\boldsymbol{\varepsilon}) \boldsymbol{\delta}+2 G \boldsymbol{\varepsilon}, \\
& \boldsymbol{\varepsilon}=\frac{1}{2}\left[\nabla \mathbf{u}+(\nabla \mathbf{u})^{\mathrm{T}}\right], \\
& \mathbf{m}=2 l^{2} G \boldsymbol{\chi}, \\
& \boldsymbol{\chi}=\frac{1}{2}\left[\nabla \boldsymbol{\theta}+(\nabla \boldsymbol{\theta})^{\mathrm{T}}\right],
\end{aligned}
$$

respectively. In the above equations, $\lambda$ and $G$ are Lamé's constants ( $G$ is also known as the shear modulus), $\delta$ is Kronecker's delta function, $l$ is a material length scale parameter capturing the size effect on the dynamic behavior, $\mathbf{u}$ is the displacement vector with the components given by (1), and $\theta$ is the rotation vector given by

$$
\boldsymbol{\theta}=\frac{1}{2} \operatorname{curl}(\mathbf{u}) \text {. }
$$

In the present paper, the geometry nonlinearity will be taken into account, so the axial strain can be expressed by the von Kármán relationship as [27]

$$
\varepsilon_{x x}=\frac{\partial u_{x}}{\partial s}+\frac{1}{2}\left(\frac{\partial w}{\partial s}\right)^{2}=\frac{\partial u}{\partial s}-z \frac{\partial \psi}{\partial s}+\frac{1}{2}\left(\frac{\partial w}{\partial s}\right)^{2} .
$$

The other nonzero components of the strain tensor can be obtained as

$$
\varepsilon_{x z}=\frac{1}{2}\left(\frac{\partial w}{\partial s}-\psi\right) .
$$

Then, we have (6) in the form of

$$
\boldsymbol{\varepsilon}=\left[\begin{array}{ccc}
\frac{\partial u}{\partial s}-z \frac{\partial \psi}{\partial s}+\frac{1}{2}\left(\frac{\partial w}{\partial s}\right)^{2} & 0 & \frac{1}{2}\left(\frac{\partial w}{\partial s}-\psi\right) \\
0 & 0 & 0 \\
\frac{1}{2}\left(\frac{\partial w}{\partial s}-\psi\right) & 0 & 0
\end{array}\right]
$$

By combining (12) and (5), one can get

$$
\boldsymbol{\sigma}=\left[\begin{array}{ccc}
{\left[\frac{\partial u}{\partial s}-z \frac{\partial \psi}{\partial s}+\frac{1}{2}\left(\frac{\partial w}{\partial s}\right)^{2}\right](\lambda+2 G)} & 0 & G\left(\frac{\partial w}{\partial s}-\psi\right) \\
0 & \lambda\left[\frac{\partial u}{\partial s}-z \frac{\partial \psi}{\partial s}+\frac{1}{2}\left(\frac{\partial w}{\partial s}\right)^{2}\right] & 0 \\
G\left(\frac{\partial w}{\partial s}-\psi\right) & 0 & \lambda\left[\frac{\partial u}{\partial s}-z \frac{\partial \psi}{\partial s}+\frac{1}{2}\left(\frac{\partial w}{\partial s}\right)^{2}\right]
\end{array}\right] .
$$


The combination of (1) and (9) yields

$$
\begin{aligned}
& \theta_{y}=-\frac{1}{2}\left(\psi+\frac{\partial w}{\partial s}\right), \\
& \theta_{x}=\theta_{z}=0 .
\end{aligned}
$$

The substitution of (14) into (8) leads to

$$
\chi=\left[\begin{array}{ccc}
0 & -\frac{1}{4}\left(\frac{\partial \psi}{\partial s}+\frac{\partial^{2} w}{\partial s^{2}}\right) & 0 \\
-\frac{1}{4}\left(\frac{\partial \psi}{\partial s}+\frac{\partial^{2} w}{\partial s^{2}}\right) & 0 & 0 \\
0 & 0 & 0
\end{array}\right] .
$$

The utilization of (7) yields

$$
\mathbf{m}=2 l^{2} G\left[\begin{array}{ccc}
0 & -\frac{1}{4}\left(\frac{\partial \psi}{\partial s}+\frac{\partial^{2} w}{\partial s^{2}}\right) & 0 \\
-\frac{1}{4}\left(\frac{\partial \psi}{\partial s}+\frac{\partial^{2} w}{\partial s^{2}}\right) & 0 & 0 \\
0 & 0 & 0
\end{array}\right] .
$$

By substituting (12), (13), (15), and (16) into equation (4), the expression of the strain energy of the pipe can be obtained as

$$
U=\frac{1}{2} \int_{\Omega}\left\{\left[\frac{\partial u}{\partial s}-z \frac{\partial \psi}{\partial s}+\frac{1}{2}\left(\frac{\partial w}{\partial s}\right)^{2}\right]^{2}(\lambda+2 G)+G\left(\frac{\partial w}{\partial s}-\psi\right)^{2}+\frac{1}{4} l^{2} G\left(\frac{\partial \psi}{\partial s}+\frac{\partial^{2} w}{\partial s^{2}}\right)^{2}\right\} \mathrm{d} v
$$

Large motions imply that terms of higher order than the linear ones have to be kept in the equation. In the current paper, although the deflection of the pipe can be considered to be large, only cubic-nonlinear terms will be retained in the final equations. Because the variational technique always requires one order higher than the one to be sought, all expressions under the integrand in (17) have to be exact to quartic-nonlinear terms. It is so for the expression of kinetic energy below.

It follows from (2) that

$$
u^{\prime}+\frac{1}{2}\left(w^{\prime}\right)^{2}=O\left(\left|w^{\prime}\right|^{4}\right)
$$

where the primes denote $\partial / \partial s$. Equation (3) can be written as

$$
w^{\prime}=\sin (\psi)=\psi-\frac{1}{3 !} \psi^{3}+O\left(|\psi|^{5}\right)
$$

which leads to

$$
\begin{aligned}
w^{\prime}-\psi & =O\left(|\psi|^{3}\right), \\
\psi & =w^{\prime}+\frac{1}{3 !}\left(w^{\prime}\right)^{3}+O\left(\left|w^{\prime}\right|^{5}\right) .
\end{aligned}
$$

After differentiating (21) with respect to $s$, we obtain

$$
\psi^{\prime}=w^{\prime \prime}+\frac{1}{2}\left(w^{\prime}\right)^{2} w^{\prime \prime}+O\left(\left|w^{\prime \prime}\right|\left|w^{\prime}\right|^{4}\right)
$$

The substitution of (18), (20), and (22) into (17) yields

$$
\begin{aligned}
U & =\frac{1}{2} \int_{\Omega}\left\{z^{2}\left(w^{\prime \prime}\right)^{2}\left[1+\left(w^{\prime}\right)^{2}\right](\lambda+2 G)+\frac{1}{4} l^{2} G\left(w^{\prime \prime}\right)^{2}\left[4+2\left(w^{\prime}\right)^{2}\right]\right\} \mathrm{d} v \\
& =\frac{1}{2} \int_{0}^{L}\left\{I\left(w^{\prime \prime}\right)^{2}\left[1+\left(w^{\prime}\right)^{2}\right](\lambda+2 G)+\frac{1}{4} A_{p} l^{2} G\left(w^{\prime \prime}\right)^{2}\left[4+2\left(w^{\prime}\right)^{2}\right]\right\} \mathrm{d} s,
\end{aligned}
$$

where $I$ is the second moment of the cross section of the pipe.

By the use of (1), the kinetic energy of the pipe can be expressed as

$$
T_{p}=\frac{1}{2} \int_{\Omega} \rho\left[\left(\frac{\partial u_{x}}{\partial t}-z \frac{\partial \psi}{\partial t}\right)^{2}+\left(\frac{\partial w}{\partial t}\right)^{2}\right] \mathrm{d} v
$$

where $\rho$ represents the mass per unit volume of the tube.

For a slender pipe, the second moment $I$ without multiplying by $E$ (i.e., the elastic modulus) or $G$ can be neglected. Additionally, the symmetry of the cross section leads to

$$
\int_{\Omega} \rho z \frac{\partial \psi}{\partial t} \frac{\partial u_{x}}{\partial t} \mathrm{~d} v=0
$$

Thus

$$
T_{p}=\frac{1}{2} \int_{\Omega} \rho\left[\left(\frac{\partial u}{\partial t}\right)^{2}+\left(\frac{\partial w}{\partial t}\right)^{2}\right] \mathrm{d} v=\frac{1}{2} m \int_{0}^{L}\left[\left(\frac{\partial u}{\partial t}\right)^{2}+\left(\frac{\partial w}{\partial t}\right)^{2}\right] \mathrm{d} s .
$$



[39]:

The kinetic energy of the fluid was given by Semler et al.

$$
T_{f}=\frac{1}{2} M \int_{0}^{L}\left\{\left[\frac{\partial u}{\partial t}+V\left(\frac{\partial u}{\partial s}+1\right)\right]^{2}+\left(\frac{\partial w}{\partial t}+V \frac{\partial w}{\partial s}\right)^{2}\right\} \mathrm{d} s .
$$

Hence, according to the formulation of Benjamin [1], Hamilton's principle for a fluid-conveying tube can be written as $\delta \int_{t_{1}}^{t_{2}}\left(T_{p}+T_{f}-U-M V^{2}\right) \mathrm{d} t-\int_{t_{1}}^{t_{2}} M V\left(\dot{w}_{L}+V w_{L}^{\prime}\right) \delta w_{L} \mathrm{~d} t=0$,

where the subscript $L$ represents the values of the corresponding quantities at $s=L$, and the dots and primes denote $\partial / \partial t$ and $\partial / \partial s$, respectively.

After several times of transformations and manipulations, the governing equation of motion is found to be

$$
\begin{aligned}
& (m+M) \ddot{w}+2 M V \dot{w}^{\prime}\left(1+w^{\prime 2}\right)+w^{\prime \prime} M V^{2}\left(1+w^{\prime 2}\right) \\
& -w^{\prime \prime}\left[\int_{s}^{L} \int_{0}^{s}(m+M)\left(\dot{w}^{\prime 2}+w^{\prime} \ddot{w}^{\prime}\right) \mathrm{d} s \mathrm{~d} s+\int_{s}^{L}\left(2 M V w^{\prime} \dot{w}^{\prime}+M V^{2} w^{\prime} w^{\prime \prime}\right) \mathrm{d} s\right] \\
& +w^{\prime} \int_{0}^{s}(m+M)\left(\dot{w}^{\prime 2}+w^{\prime} \ddot{w}^{\prime}\right) \mathrm{d} s+\operatorname{EI}\left(w^{(4)}+4 w^{\prime} w^{\prime \prime} w^{\prime \prime \prime}+w^{\prime \prime 3}+w^{(4)} w^{\prime 2}\right) \\
& +A_{p} l^{2} G\left(w^{(4)}+2 w^{\prime} w^{\prime \prime} w^{\prime \prime \prime}+\frac{1}{2} w^{\prime \prime 3}+\frac{1}{2} w^{(4)} w^{\prime 2}\right)=0,
\end{aligned}
$$

where the Poisson effect is neglected, and $(\lambda+2 G) I$ is then replaced by EI.

By assuming that the internal dissipation of the pipe material is viscoelastic and of the Kelvin-Voigt type with coefficient $a$, according to the analysis given by Semler et al.
[39], we only need to add this term $a \mathrm{EI} \dot{w}^{(4)}$ to (29) to take the dissipation into account.

Furthermore, with the introduction of the impact force modeled as a cubic spring with stiffness $K$, that is, $f(w)=$ $K w^{3}[12]$, the resulting governing equation of motion can be written as

$$
\begin{aligned}
& (m+M) \ddot{w}+2 M V \dot{w}^{\prime}\left(1+w^{\prime 2}\right)+w^{\prime \prime} M V^{2}\left(1+w^{\prime 2}\right) \\
& -w^{\prime \prime}\left[\int_{s}^{L} \int_{0}^{s}(m+M)\left(\dot{w}^{\prime 2}+w^{\prime} \ddot{w}^{\prime}\right) \mathrm{d} s \mathrm{~d} s+\int_{s}^{L}\left(2 M V w^{\prime} \dot{w}^{\prime}+M V^{2} w^{\prime} w^{\prime \prime}\right) \mathrm{d} s\right] \\
& +w^{\prime} \int_{0}^{s}(m+M)\left(\dot{w}^{\prime 2}+w^{\prime} \ddot{w}^{\prime}\right) \mathrm{d} s+\mathrm{EI}\left(w^{(4)}+4 w^{\prime} w^{\prime \prime} w^{\prime \prime \prime}+w^{\prime \prime 3}+w^{(4)} w^{\prime 2}\right) \\
& +A_{p} l^{2} G\left(w^{(4)}+2 w^{\prime} w^{\prime \prime} w^{\prime \prime \prime}+\frac{1}{2} w^{\prime \prime 3}+\frac{1}{2} w^{(4)} w^{\prime 2}\right) \\
& +a \mathrm{EI} \dot{w}^{(4)}+K w^{3} \delta\left(s-s_{b}\right)=0 .
\end{aligned}
$$


By introducing the following nondimensional quantities, one can write (30) in its dimensionless form as follows:

$$
\begin{aligned}
& \eta=\frac{w}{L} \\
& \xi=\frac{s}{L} \\
& \tau=\left[\frac{\mathrm{EI}}{(m+M) L^{4}}\right]^{1 / 2} t \\
& v=\left(\frac{M}{\mathrm{EI}}\right)^{1 / 2} V L \\
& \beta=\frac{M}{M+m}, \\
& \kappa=\frac{\mathrm{KL}^{5}}{\mathrm{EI}}, \\
& \xi_{b}=\frac{s_{b}}{L}, \\
& \alpha=\left[\frac{\mathrm{EI}}{(m+M) L^{4}}\right]^{1 / 2} a, \\
& \ddot{\eta}+2 v \sqrt{\beta} \dot{\eta}^{\prime}\left(1+\eta^{\prime 2}\right)+v^{2} \eta^{\prime \prime}\left(1+\eta^{\prime 2}\right) \\
& -\eta^{\prime \prime}\left[\int_{\xi}^{1} \int_{0}^{\xi}\left(\dot{\eta}^{\prime 2}+\eta^{\prime} \ddot{\eta}^{\prime}\right) \mathrm{d} \xi \mathrm{d} \xi+\int_{\xi}^{1}\left(2 v \sqrt{\beta} \eta^{\prime} \dot{\eta}^{\prime}+v^{2} \eta^{\prime} \eta^{\prime \prime}\right) \mathrm{d} \xi\right] \\
& +\eta^{\prime} \int_{0}^{\xi}\left(\dot{\eta}^{\prime 2}+\eta^{\prime} \ddot{\eta}^{\prime}\right) \mathrm{d} \xi+\left(\eta^{(4)}+4 \eta^{\prime} \eta^{\prime \prime} \eta^{\prime \prime \prime}+\eta^{\prime \prime 3}+\eta^{(4)} \eta^{\prime 2}\right) \\
& +\frac{A_{p} l^{2} G}{\mathrm{EI}}\left(\eta^{(4)}+2 \eta^{\prime} \eta^{\prime \prime} \eta^{\prime \prime \prime}+\frac{1}{2} \eta^{\prime \prime 3}+\frac{1}{2} \eta^{(4)} \eta^{\prime 2}\right)+\alpha \dot{\eta}^{(4)}+\kappa \eta^{3} \delta\left(\xi-\xi_{b}\right)=0 .
\end{aligned}
$$

It is observed that there exists two nonlinear inertial terms in (32), which are the same as in $[39,41]$. The appearance of nonlinear inertial terms render the equation nonstandard and call the straightforward application of conventional dynamic system theory into question. Because $\eta$ represents the lateral displacement divided by the length $L$, $\eta$ could be assumed to be small. Following the approach used by Li and Paidoussis [41] and Semler et al. [39], the nonlinear inertial terms can be eliminated through a perturbation technique, by which (32) can be reduced to the form without nonlinear inertial terms:

$$
\begin{aligned}
\ddot{\eta}+ & 2 v \sqrt{\beta} \dot{\eta}^{\prime}+v^{2} \eta^{\prime \prime}+\left(1+2 l_{0}\right) \eta^{(4)}+\alpha \dot{\eta}^{(4)}+\kappa \eta^{3} \delta\left(\xi-\xi_{b}\right) \\
& +2 v \sqrt{\beta} \dot{\eta}^{\prime} \eta^{\prime 2}+v^{2} \eta^{\prime \prime} \eta^{\prime 2}+\left(3+2 l_{0}\right) \eta^{\prime} \eta^{\prime \prime} \eta^{\prime \prime \prime}+\left(1+l_{0}\right) \eta^{\prime \prime 3}-l_{0} \eta^{(4)} \eta^{\prime 2} \\
& +\eta^{\prime} \int_{0}^{\xi}\left[\dot{\eta}^{\prime 2}-2 v \sqrt{\beta} \dot{\eta}^{\prime \prime} \eta^{\prime}-v^{2} \eta^{\prime} \eta^{\prime \prime \prime}+\left(1+2 l_{0}\right) \eta^{\prime \prime} \eta^{(4)}\right] \mathrm{d} \xi \\
& -\eta^{\prime \prime} \int_{\xi}^{1}\left[2 v \sqrt{\beta} \eta^{\prime} \dot{\eta}^{\prime}+v^{2} \eta^{\prime} \eta^{\prime \prime}+\left(1+2 l_{0}\right) \eta^{\prime \prime} \eta^{\prime \prime \prime}\right] \mathrm{d} \xi \\
& -\eta^{\prime \prime} \int_{\xi}^{1} \int_{0}^{\xi}\left[\dot{\eta}^{\prime 2}-2 v \sqrt{\beta} \dot{\eta}^{\prime \prime} \eta^{\prime}-v^{2} \eta^{\prime} \eta^{\prime \prime \prime}+\left(1+2 l_{0}\right) \eta^{\prime \prime} \eta^{(4)}\right] \mathrm{d} \xi \mathrm{d} \xi=0 .
\end{aligned}
$$


In the above equation, we let $l_{0}=(1 / 2)\left(A_{p} l^{2} G / \mathrm{EI}\right)$ for simplicity.

\section{Discretization of the Governing Equation}

By letting

$$
\eta(\xi, \tau)=\sum_{r=1}^{N} \varphi_{r}(\xi) q_{r}(\tau)
$$

where

$$
\begin{array}{r}
\varphi_{r}(\xi)=\cos h \lambda_{r} \xi-\cos \lambda_{r} \xi-\sigma_{r}\left(\sin h \lambda_{r} \xi-\sin \lambda_{r} \xi\right), \\
\sigma_{r}=\frac{\left(\sin h \lambda_{r}-\sin \lambda_{r}\right)}{\left(\cos h \lambda_{r}+\cos \lambda_{r}\right)}, \quad(r=1,2, \ldots, N),
\end{array}
$$

$\varphi_{r}(\xi)$ is the eigenfunction of the cantilevered beam, $q_{r}(\tau)$ is the corresponding generalized coordinate, and $\lambda_{r}$ is the beam eigenvalue. According to Galerkin's technique, after substituting (34) into (33), multiplication by the factor $\varphi_{r}(\xi)$ followed by integration from 0 to 1 leads to [12]

$$
\ddot{q}_{i}+c_{i j} \dot{q}_{j}+k_{i j} \dot{q}_{j}+\alpha_{i j k l} q_{j} q_{k} q_{l}+\beta_{i j k l} q_{j} q_{k} \dot{q}_{l}+\gamma_{i j k l} q_{j} \dot{q}_{k} \dot{q}_{l}=0,
$$

where $\quad c_{i j}=\delta_{i j} \alpha \lambda_{i}^{4}+2 v \sqrt{\beta} \int_{0}^{1} \phi_{i} \phi_{j}^{\prime} \mathrm{d} \xi, \quad k_{i j}=\delta_{i j}\left(1+2 l_{0}\right)$ $\int_{0}^{1} \phi_{i}^{(4)} \phi_{j} \mathrm{~d} \xi+v^{2} \int_{0}^{1} \phi_{i} \phi_{j}^{\prime \prime} \mathrm{d} \xi, \quad \alpha_{i j k l}=\int_{0}^{1} \phi_{i} \cdot\left[l_{0}\left(2 \phi_{j}^{\prime} \phi_{k}^{\prime \prime} \phi_{k}^{\prime \prime}+\right.\right.$ $\phi_{j}^{\prime \prime} \phi_{k}^{\prime \prime} \phi_{l}^{\prime \prime}-\phi_{j}^{\prime} \phi_{k}^{\prime} \phi_{l}^{\prime \prime}+2 \phi_{j}^{\prime} \int_{0}^{\xi} \phi_{k}^{\prime \prime} \phi_{l}^{(4)} \mathrm{d} \xi-2 \phi_{j}^{\prime \prime} \quad \int_{\xi}^{1} \phi_{k}^{\prime \prime} \phi_{l}^{\prime \prime} \mathrm{d} \xi-2 \phi_{j}^{\prime \prime}$ $\left.\int_{\xi}^{1} \int_{0}^{\xi} \phi_{k}^{\prime \prime} \quad \phi_{l}^{(4)} \mathrm{d} \xi \mathrm{d} \xi\right)+v^{2}\left(\phi_{j}^{\prime} \phi_{k}^{\prime} \phi_{l}^{\prime \prime}-\phi_{j}^{\prime} \int_{0}^{\xi} \phi_{k}^{\prime} \phi_{l}^{\prime \prime} \mathrm{d} \xi-\phi_{j}^{\prime \prime}\right.$ $\left.\int_{\xi}^{1} \phi_{k}^{\prime} \phi_{l}^{\prime \prime} \mathrm{d} \xi+\phi_{j}^{\prime \prime} \int_{\xi}^{1} \int_{0}^{\xi} \phi_{k}^{\prime} \phi_{l}^{\prime \prime} \mathrm{d} \xi \mathrm{d} \xi\right)+3 \phi_{j}^{\prime} \phi_{k}^{\prime \prime} \phi_{l}^{\prime \prime}+\phi_{j}^{\prime \prime} \phi_{k}^{\prime \prime} \phi_{l}^{\prime \prime}+$ $\left.\phi_{j}^{\prime} \int_{0}^{\xi} \phi_{k}^{\prime \prime} \phi_{l}^{(4)} \mathrm{d} \xi-\phi_{j}^{\prime \prime} \int_{\xi}^{1} \phi_{k}^{\prime \prime} \phi_{l}^{\prime \prime} \mathrm{d} \xi-\phi_{j}^{\prime \prime} \int_{\xi}^{1} \int_{0}^{\xi} \phi_{k}^{\prime \prime} \phi_{l}^{(4)} \mathrm{d} \xi \mathrm{d} \xi\right] \mathrm{d} \xi$, $\beta_{i j k l}=\int_{0}^{1} \phi_{i} \cdot 2 v \sqrt{\beta}\left(\phi_{j}^{\prime} \phi_{k}^{\prime} \phi_{l}^{\prime}-\phi_{j}^{\prime} \int_{0}^{\xi} \phi_{k}^{\prime} \phi_{l}^{\prime \prime} d \xi-\phi_{j}^{\prime \prime} \int_{\xi}^{1} \phi_{k}^{\prime} \phi_{l}^{\prime} d \xi+\right.$ $\left.\phi_{j}^{\prime \prime} \quad \int_{\xi}^{1} \int_{0}^{\xi} \phi_{k}^{\prime} \phi_{l}^{\prime \prime} d \xi d \xi\right) d \xi, \quad \gamma_{i j k l}=\int_{0}^{1} \phi_{i} \cdot\left(\phi_{j}^{\prime} \int_{0}^{\xi} \phi_{k}^{\prime} \phi_{l}^{\prime} \mathrm{d} \xi-\right.$ $\left.\phi_{j}^{\prime \prime} \int_{\xi}^{1} \int_{0}^{\xi} \phi_{k}^{\prime} \phi_{l}^{\prime} \mathrm{d} \xi \mathrm{d} \xi\right) \mathrm{d} \xi$, where the dots and primes represent $\partial / \partial \tau$ and $\partial / \partial \xi$, respectively, and the indices $i, j, k$, and $l$ range from 1 to $N$.

As shown by Paidoussis and Semler [12], the dynamics in the two-mode version of the analytical model is in good qualitative agreement with the experimental observations. Since the main purpose of this paper is to investigate part of the qualitative behaviors of the present system, the two-mode expansion, that is, $N=2$ in (34), is adopted to discretize (33).

In order to apply the available tools from dynamic system theory, we render (36) into the first-order form by introducing the following transformations:

$$
\begin{aligned}
& q_{1}=x_{1}, \\
& q_{2}=x_{2}, \\
& \dot{q}_{1}=x_{3}, \\
& \dot{q}_{2}=x_{4} .
\end{aligned}
$$

Equation (36) can be rewritten in matrix form as

$$
\dot{X}=\mathbf{L X}+\mathbf{N}(\mathbf{X}) \text {, }
$$

where

$$
\mathbf{L}=\left[\begin{array}{cccc}
0 & 0 & 1 & 0 \\
0 & 0 & 0 & 1 \\
-k_{11} & -k_{12} & -c_{11} & -c_{12} \\
-k_{21} & -k_{22} & -c_{21} & -c_{22}
\end{array}\right],
$$

$\mathbf{N}(\mathbf{X})$ represents the cubic-nonlinear terms of (36).

\section{Theoretical Analysis and Numerical Computations}

It should be noted that $\mathbf{X}=(0,0,0,0)$ is always a solution to (38). The equilibrium is stable for small internal flow velocity and may lose stability when the flow velocity crossed a critical value. As will be shown below, the pipe becomes unstable by flutter and the size effect on the critical velocity is significant. The present work mainly investigates the effect of the internal material length scale parameter on the dynamic behaviors of micropipes and the bifurcation diagrams with increasing flow velocity. Hence, among the five dimensionless quantities $v, \beta, \kappa, \xi_{b}, \alpha$ in (32) and the dimensionless quantity $l_{0}$ in (33), only $v$ and $l_{0}$ are considered to be variable parameters. In what follows, we choose the values of the fixed parameters to be

$$
\begin{aligned}
\alpha & =0.005, \\
\beta & =0.2, \\
\xi_{b} & =0.82, \\
\kappa & =10^{5},
\end{aligned}
$$

as was also utilized in [12]. $v$ and $l_{0}$ are kept as controllable parameters for the determination of critical flow velocity. We study first the loss of stability of the equilibrium, that is, the occurrence of the Hopf bifurcation. Based on the numerical simulation, the graph of $v$ versus $l_{0}$, representing the size effect on the critical velocity, is shown in Figure 2. It can be seen that by increasing the value of $l_{0}$, the critical flow velocity that was predicted from the modified couple stress theory increases.

For each point $\left(\widetilde{l}_{0}, \widetilde{v}\right)$ of the curve, the Jacobian matrix of (38) at the equilibrium possesses a pair of pure imaginary eigenvalues and two eigenvalues with negative real part, known as a degenerate point. Up to this point, it is potential for the Hopf bifurcation to occur. Technically, we need to verify another two inequalities, that is, $\mu_{\varepsilon}^{\prime}(0) \neq 0$ and $l_{1}(0) \neq 0$, called the "transversality" and "the first Lyapunov's coefficient not being zero," respectively, to complete the analysis, where $\mu_{\varepsilon}^{\prime}(0)$ and $l_{1}(0)$ will be defined below.

Physically, for a given microtube, $l_{0}$ is undoubtedly a constant, but $v$ remains a controllable parameter. Hence, it is convenient to write (38) near a degenerate point as

$$
\dot{X}=\mathbf{A}\left(\widetilde{l}_{0}, \widetilde{v}+\varepsilon\right) \mathbf{X}+\mathbf{F}\left(\mathbf{X}, \tilde{l}_{0}, \widetilde{v}+\varepsilon\right),
$$

where $\varepsilon$ represents the perturbation of the critical velocity $\widetilde{v}$.

Letting $\lambda(\varepsilon)=\mu(\varepsilon) \pm i \omega_{0}(\varepsilon)$ be the eigenvalues of matrix $\mathbf{A}\left(\tilde{l}_{0}, \widetilde{v}+\varepsilon\right)$ satisfying $\lambda(0)= \pm \mathrm{i} \omega_{0}$ with $\omega_{0}>0 . \mathbf{q}(\varepsilon)$ and $\mathbf{p}(\varepsilon)$ are chosen as being defined by 


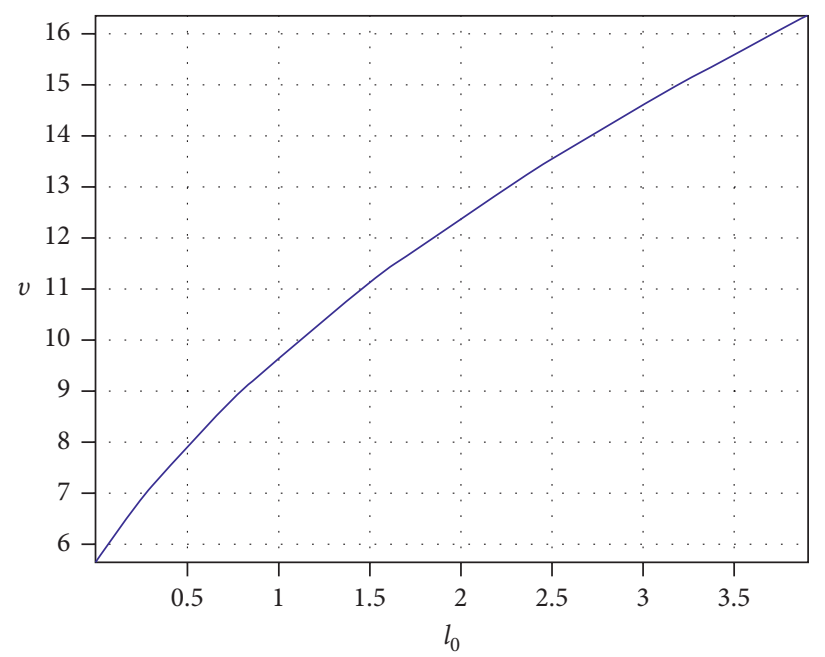

Figure 2: For the system defined by (38) with $\alpha=0.005, \beta=0.2, \xi_{b}=0.82, \kappa=10^{5}$,the critical flow velocity versus the nondimensional quantity $l_{0}$ defined by $l_{0}=A_{p} l^{2} G /(2 \mathrm{EI})$.

$$
\begin{aligned}
\mathbf{A}\left(\tilde{l}_{0}, \widetilde{v}+\varepsilon\right) \mathbf{q}(\varepsilon) & =\lambda(\varepsilon) \mathbf{q}(\varepsilon), \\
\mathbf{A}^{\mathrm{T}}\left(\widetilde{l}_{0}, \widetilde{v}+\varepsilon\right) \mathbf{p}(\varepsilon) & =\bar{\lambda}(\varepsilon) \mathbf{p}(\varepsilon), \\
<\mathbf{p}(\varepsilon), \mathbf{q}(\varepsilon)> & =1,
\end{aligned}
$$

where $\langle\cdot, \cdot\rangle$ represents the scalar product defined by $<\mathbf{p}, \mathbf{q}>=\bar{p}^{\mathrm{T}} \mathbf{q}$.

By differentiating $(42 \mathrm{a})$ with respect to $\varepsilon$, we find that $\mathbf{A}_{\varepsilon}^{\prime}\left(\widetilde{l}_{0}, \widetilde{v}\right) \mathbf{q}(0)+\mathbf{A}\left(\widetilde{l}_{0}, \widetilde{v}\right) \mathbf{q}_{\varepsilon}^{\prime}(0)=\lambda_{\varepsilon}^{\prime}(0) \mathbf{q}(0)+\lambda(0) \mathbf{q}_{\varepsilon}^{\prime}(0)$.

By multiplying by $\bar{p}(0)^{\mathrm{T}}$, one obtains

$$
\lambda_{\varepsilon}^{\prime}(0)=\left\langle\mathbf{p}(0), \mathbf{A}_{\varepsilon}^{\prime}\left(\widetilde{l}_{0}, \widetilde{v}\right) \mathbf{q}(0)>.\right.
$$

Thus

$$
\mu_{\varepsilon}^{\prime}(0)=\operatorname{Re}<\mathbf{p}(0), \mathbf{A}_{\varepsilon}^{\prime}\left(\widetilde{l}_{0}, \widetilde{v}\right) \mathbf{q}(0)>.
$$

The calculation of $l_{1}(0)$ is related to two aspects: one associated with the center manifold theory and the other associated with the normal form method. In what follows, we only give the formulation of $l_{1}(0)$. For more details on this topic, the interested reader is referred to [42, 43]. For this purpose, we write (41) with $\varepsilon=0$ as

$$
\begin{aligned}
\dot{X}= & \mathbf{A}\left(\tilde{l}_{0}, \widetilde{v}\right) \mathbf{X}+\mathbf{F}\left(\mathbf{X}, \tilde{l}_{0}, \widetilde{v}\right)=\mathbf{A}\left(\tilde{l}_{0}, \widetilde{v}\right) \mathbf{X}+\frac{1}{2} \mathbf{B}\left(\mathbf{X}, \mathbf{X}, \widetilde{l}_{0}, \widetilde{v}\right) \\
& +\frac{1}{3 !} \mathbf{C}\left(\mathbf{X}, \mathbf{X}, \mathbf{X}, \tilde{l}_{0}, \widetilde{v}\right)
\end{aligned}
$$

where $\mathbf{B}\left(\mathbf{X}, \mathbf{X}, \widetilde{l}_{0}, \widetilde{v}\right)$ and $\mathbf{C}\left(\mathbf{X}, \mathbf{X}, \mathbf{X}, \widetilde{l}_{0}, \widetilde{v}\right)$ are the symmetric multilinear vector functions given by

$$
\begin{gathered}
B_{i}\left(\mathbf{X}, \mathbf{X}, \widetilde{l}_{0}, \widetilde{v}\right)=\left.\sum_{j, k=1}^{4} \frac{\partial^{2} F_{i}\left(\mathbf{Y}, \widetilde{l}_{0}, \widetilde{v}\right)}{\partial y_{j} \partial y_{k}}\right|_{\mathbf{Y}=0} x_{j} x_{k}, \quad(i=1,2,3,4), \\
C_{i}\left(\mathbf{X}, \mathbf{X}, \mathbf{X}, \widetilde{l}_{0}, \widetilde{v}\right)=\left.\sum_{j, k, l=1}^{4} \frac{\partial^{3} F_{i}\left(\mathbf{Y}, \tilde{l}_{0}, \widetilde{v}\right)}{\partial y_{j} \partial y_{k} \partial y_{l}}\right|_{\mathbf{Y}=0} x_{j} x_{k} x_{l}, \quad(i=1,2,3,4),
\end{gathered}
$$

respectively.

For the symmetry of the current system, we have $\mathbf{B}\left(\mathbf{X}, \mathbf{X}, \widetilde{l}_{0}, \widetilde{v}\right)=\mathbf{0}$.

The utilization of the projection method [42] leads to

$$
l_{1}(0)=\frac{1}{2 \omega_{0}} \operatorname{Re}<\mathbf{p}(0), \mathbf{C}\left(\mathbf{q}(0), \mathbf{q}(0), \bar{q}(0), \tilde{l}_{0}, \widetilde{v}\right)>\text {. }
$$

With the aid of a computer, the plots of $\mu \prime(0)$ and $l_{1}(0)$ that are associated with each value of $\left(\widetilde{l}_{0}, \widetilde{v}\right)$ on the curve in Figure 2 can be obtained, as shown in Figures 3 and 4.
As can be seen from the two figures above, $\mu \prime(0)$ and $l(0)$ are not equal to zero and then the Hopf bifurcation can take place. The stability of this bifurcated solution depends on the sign of $l_{1}(0)$. The periodic solution is stable in case $l_{1}(0)$ is negative while it is unstable when $l_{1}(0)$ is positive, as said to be "supercritical" and "subcritical" bifurcation, respectively. A variant of the macrotube system that was modified to contain a "friction factor" was studied by Bajaj et al. [11], and the subcritical Hopf bifurcation might occur for certain regions of the parameter space. For the present system, it 


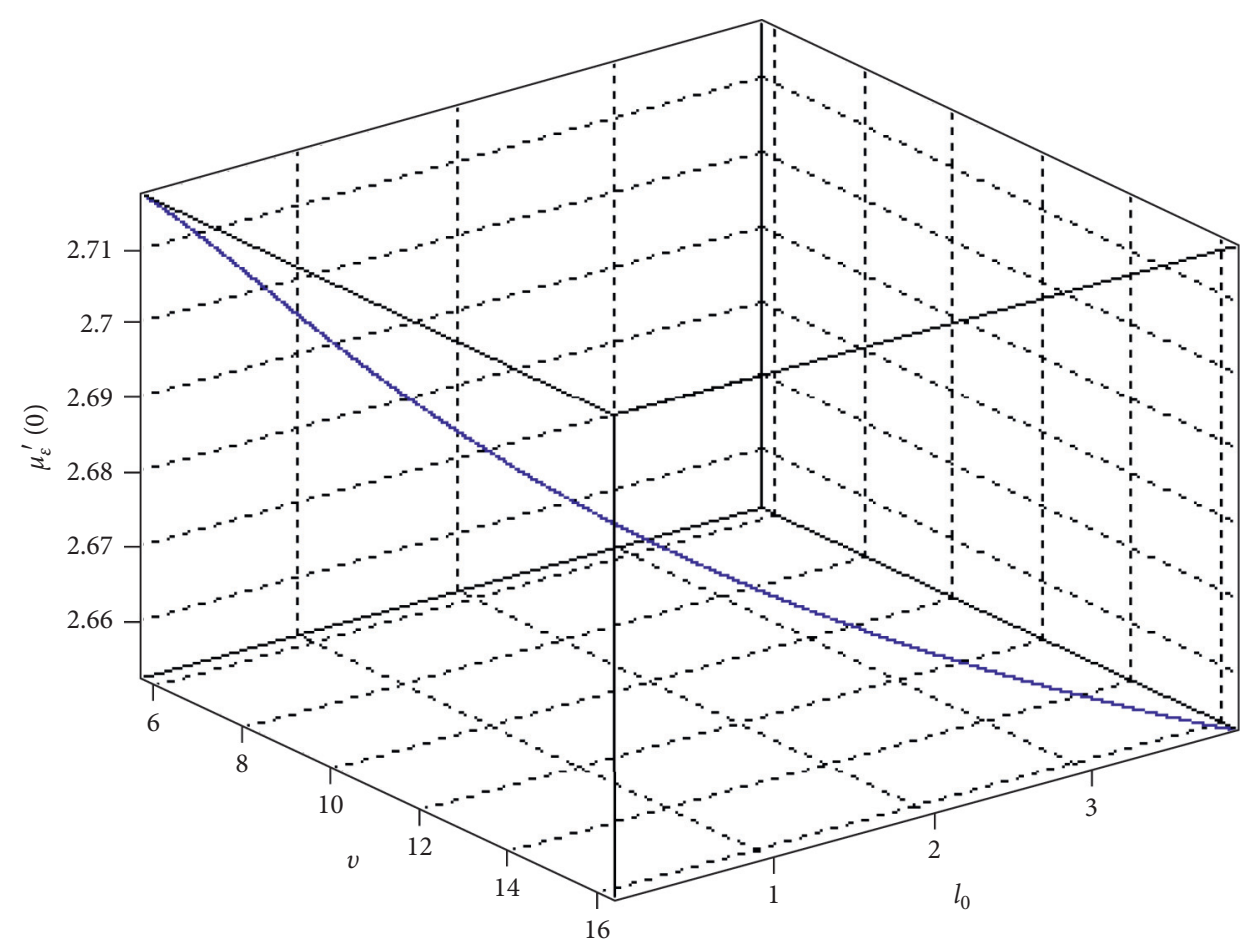

FIGURE 3: For the system of Figure 2, the curve of $\mu_{\varepsilon}^{\prime}(0)$ associated with each point $\left(\widetilde{l}_{0}, \widetilde{v}\right)$.

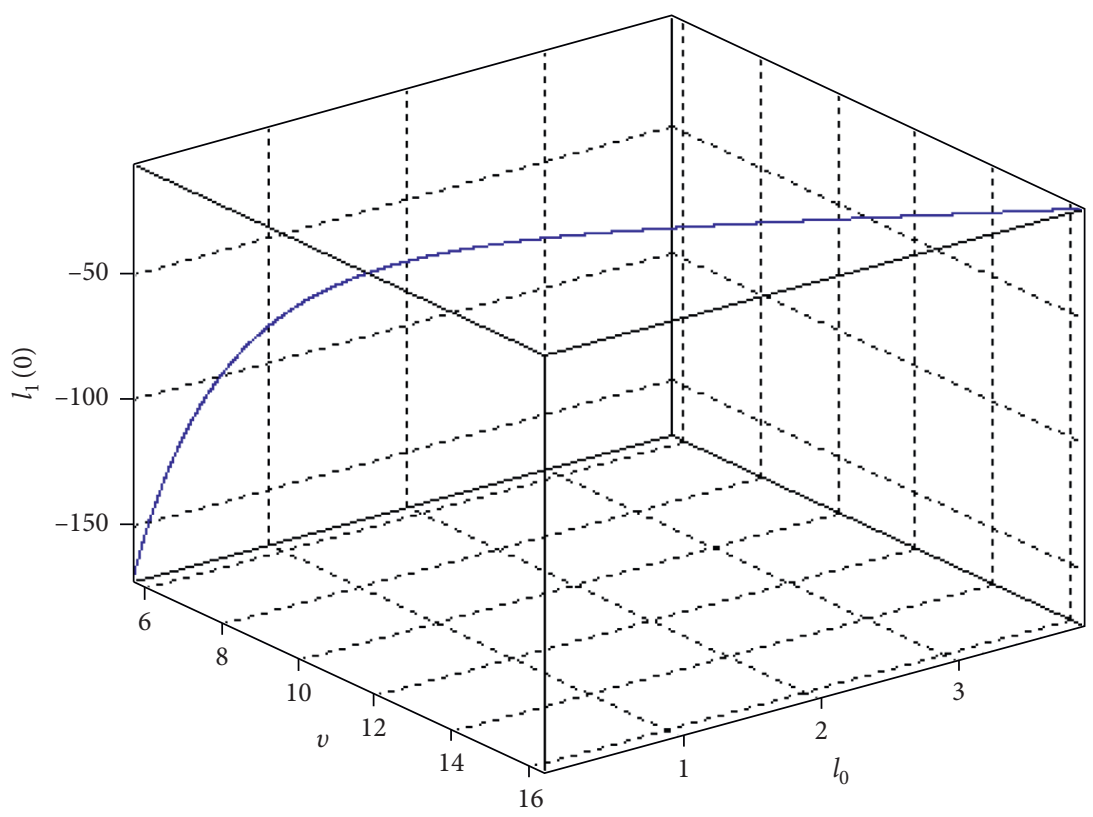

Figure 4: For the system of Figure 2, the values of the first Lyapunov's coefficient $l_{1}(0)$ at each point $\left(\widetilde{l}_{0}, \widetilde{v}\right)$.

should be noted that $l_{1}(0)$ is always negative and then the Hopf bifurcation is supercritical. In what follows, the dynamics will be explored numerically.

For various values of $l_{0}$, that is, at $0,0.2,0.5$, and 1 , the calculations have produced the bifurcation diagrams shown in Figures 5-8. In these figures the displacement plotted in the ordinate is the amplitude $x_{1}$ and the variable parameter is the dimensionless flow velocity $v$. Having discarded the transient solutions, whenever the velocity $x_{4}$ was zero, we recorded the displacement $x_{1}$, constructing the bifurcation diagrams with positive and negative values, as shown in Figures 5-8. It should be mentioned that the model will reduce to the classical tube model when the size effect is suppressed by letting $l_{0}=0$.

In these figures, it is observed that the qualitative dynamics of the system is similar to each other. The Hopf 


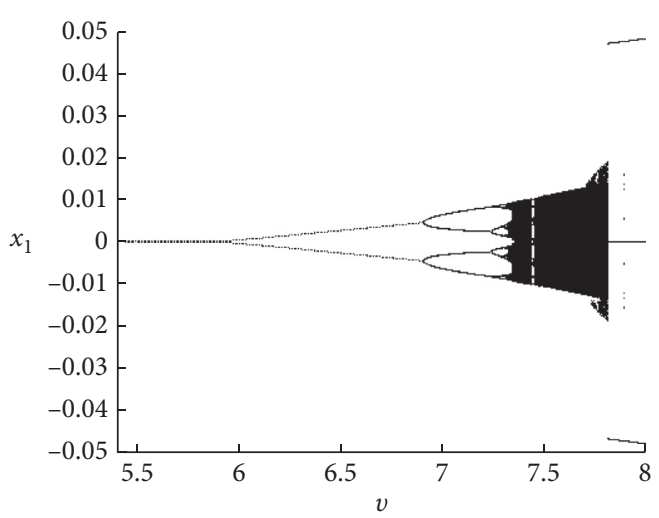

(a)

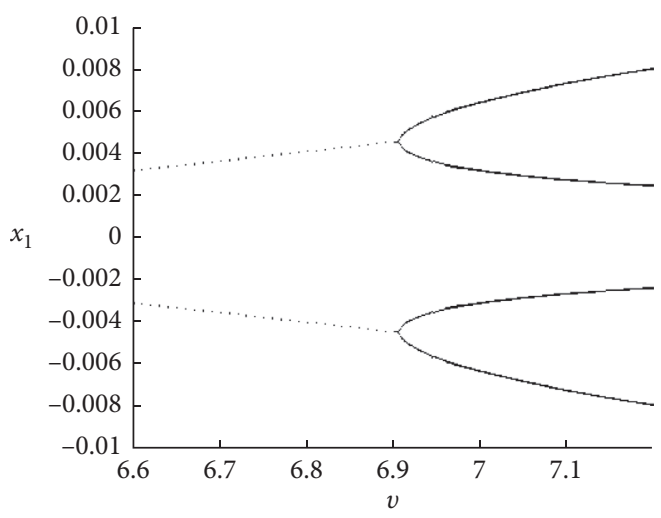

(c)

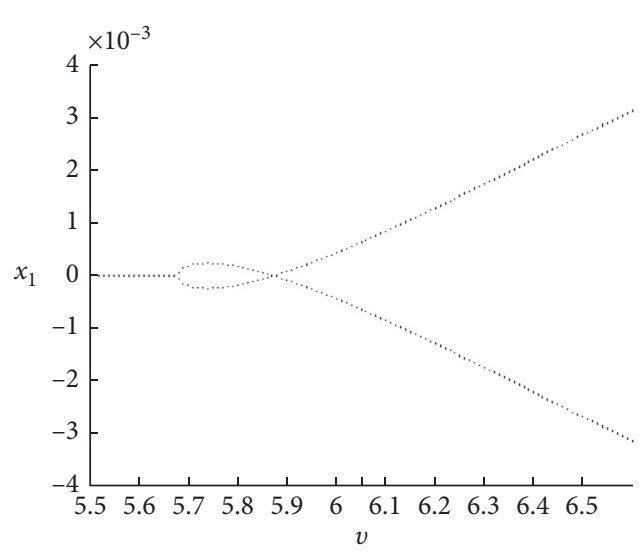

(b)

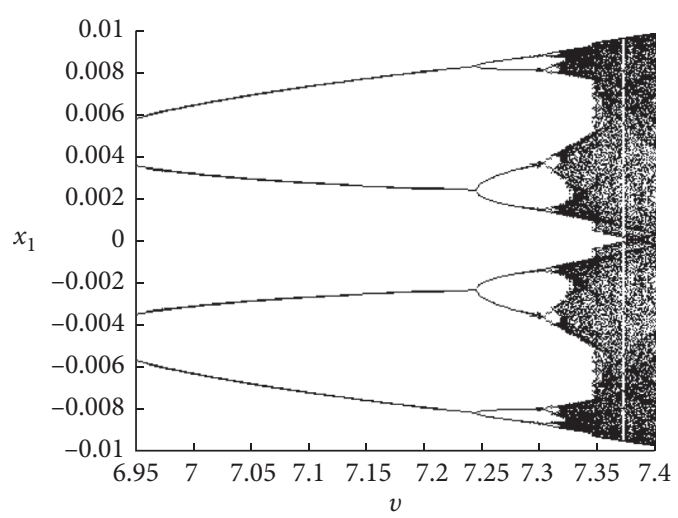

(d)

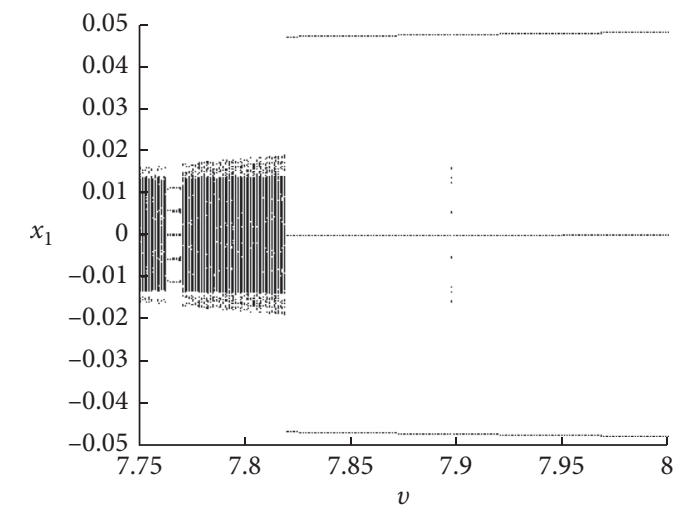

(e)

Figure 5: The bifurcation diagrams of the nonlinear system defined by (38) for $\alpha=0.005, \beta=0.2, \xi_{b}=0.82, \kappa=10^{5}$, and $l_{0}=0$, as $v$ is varied. (a) For $5.4 \leq v \leq 8$ and (b), (c), (d), and (e) for smaller ranges of $v$ to show finer details.

bifurcation occurs first and then the pipe undergoes limit cycle motion with symmetric trajectory, which is followed by a pitchfork bifurcation thereafter the limit cycle lost its symmetry. As $v$ increases further, a sequence of perioddoubling bifurcation arises, leading the motions to chaos. Beyond the chaotic band, the system reverts to the periodic motion of period 3 . From the point of view of singularity theory, in spite of the different physical assumptions made for the system, when neglecting the terms due to gravity and introducing the material length scale parameter $l$, the pipe behaviors are qualitatively like those of Paidoussis et al. [8] as well as Paidoussis and Semler [12]. In fact, the similar bifurcation sequence can be also found in other vibroimpact system with symmetry [44]. However, for the reliable and optical design of microtube, it is vital for us to distinguish critical flow velocity $v$, the threshold of chaotic motion, and so forth, for different values of $l_{0}$. We then compare these bifurcation diagrams in order to explore the effect of the parameter $l_{0}$ on the system under consideration. 


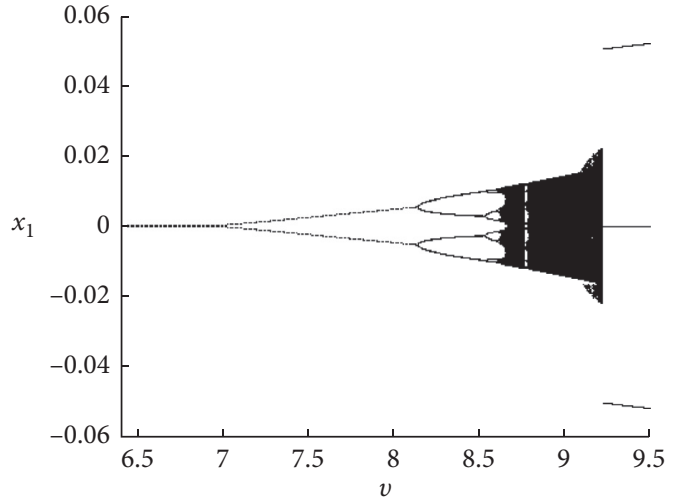

(a)

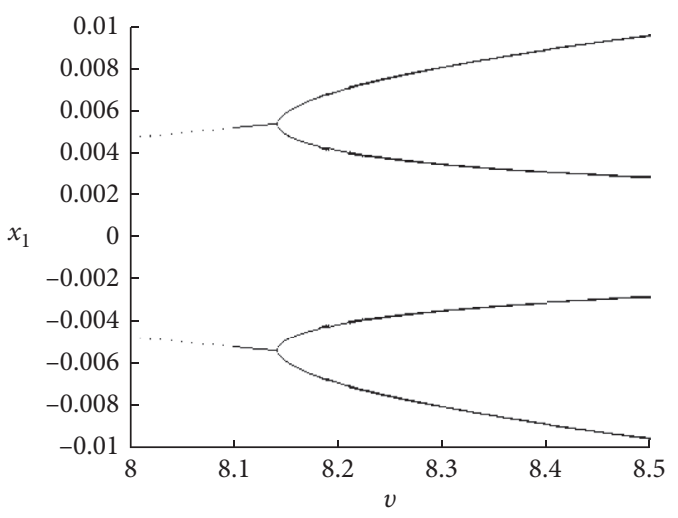

(c)

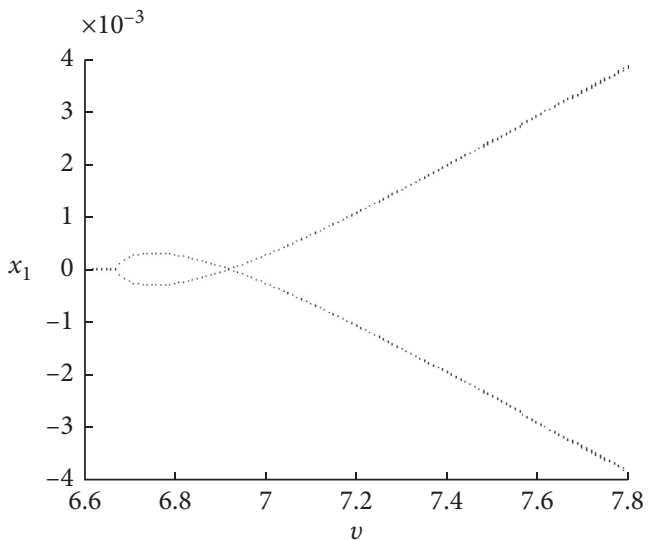

(b)

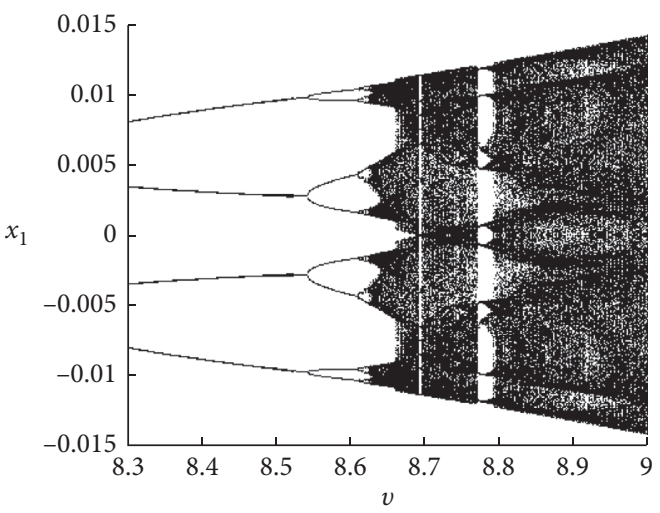

(d)

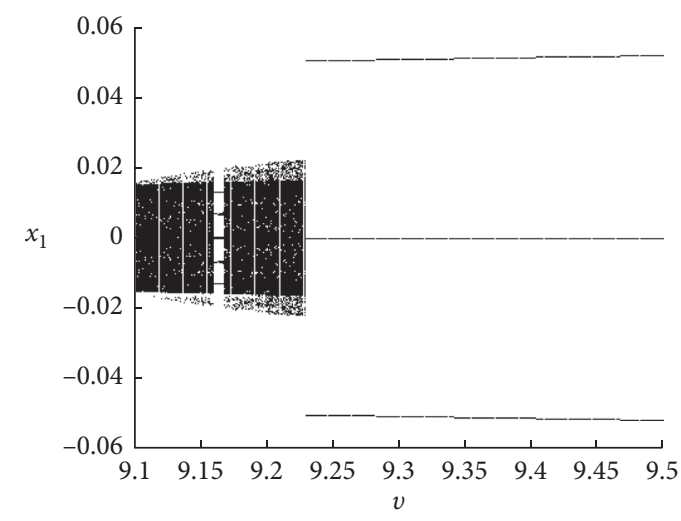

(e)

Figure 6: The bifurcation diagrams of the nonlinear system defined by (38) for $\alpha=0.005, \beta=0.2, \xi_{b}=0.82, \kappa=10^{5}$, and $l_{0}=0.2$, as $v$ is varied. (a) For $6.4 \leq v \leq 9.5$ and (b), (c), (d), and (e) for smaller ranges of $v$, showing more details.

For brevity, we only choose two figures, corresponding to $l_{0}=0$ and $l_{0}=0.2$, respectively, to complete this analysis. For this purpose, it is convenient to amplify these two bifurcation diagrams over a smaller range of $v$ as shown in Figures 5(b)-5(e) and Figures 6(b)-6(e). Thus, the finer details can be observed. The Hopf bifurcation is noted at $v=5.6784138$ for $l_{0}=0$, while it was noted at $v=6.6766354$, a higher flow velocity, for $l_{0}=0.2$. A series of $v$, that is,
$6.905075,7.245702,7.303842,7.316114$, and so forth, at which the pitchfork bifurcation and a sequence of perioddoubling bifurcations occur for the case of $l_{0}=0$, is lower than that for $l_{0}=0.2$, that is, $8.141000,8.545417,8.614248$, 8.628766 , and so forth. It is so for the occurrence of chaos and the resumption of periodic motions. Based on the results, it seems that the internal material length scale parameter makes the microtube more stable. 


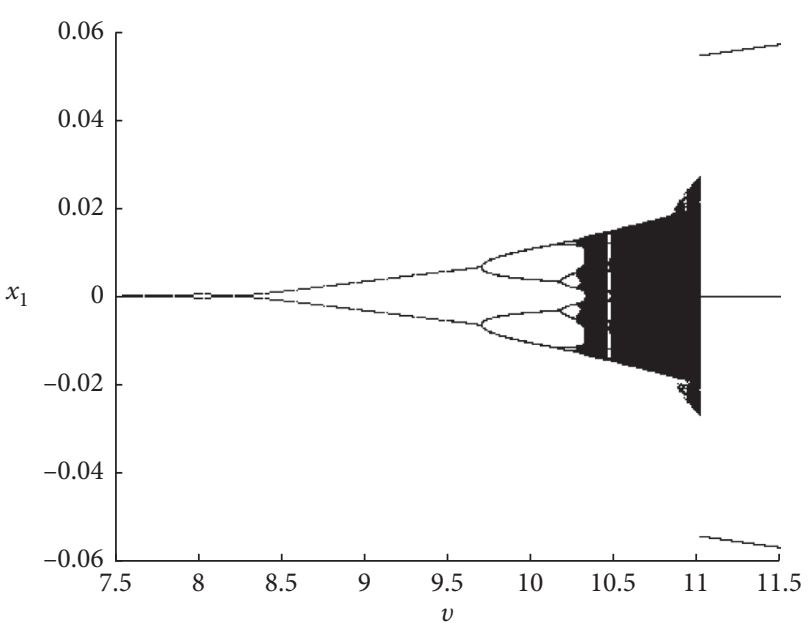

FIgURE 7: The bifurcation diagram for the nonlinear system of Figure 6 with $l_{0}=0.5$, as $v$ is varied.

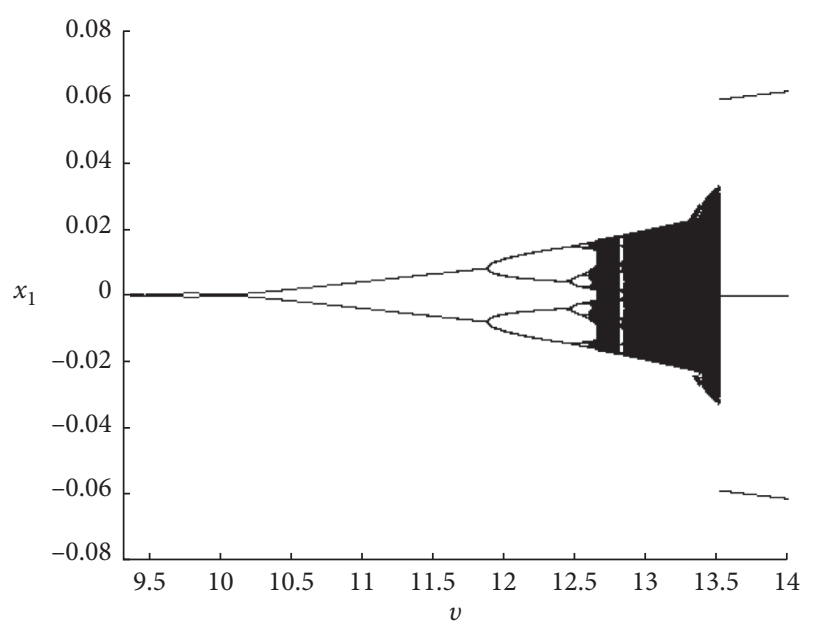

FIgURE 8: The bifurcation diagram for the nonlinear system of Figure 7 with $l_{0}=1$, as $v$ is varied.

\section{Conclusions}

In this paper, the full nonlinear equation of a fluidconveying cantilevered microtube undergoing large motions and subjected to motion-limiting constraints was deduced based on the Hamilton principle and the modified couple stress theory. The governing equation of motion was discretized into a set of ordinary differential equations via Galerkin's method. The effect of the internal material length scale parameter on the critical flow velocity was investigated. By using the projection method, the supercritical Hopf bifurcation was demonstrated. The results show that the size effect on the vibration properties is significant. The following outcomes were found from the results:

(i) The size effect on the governing equation, especially on the nonlinear terms in the governing equation was obtained. (ii) The results showed that, by increasing the value of the nondimensional internal material length scale parameter $l_{0}$, the critical flow velocity $v$ predicted from the modified couple stress theory increases.

(iii) At each degenerate point $\left(\widetilde{l}_{0}, \widetilde{v}\right)$ of the curve of the critical flow velocity versus the nondimensional quantity $l_{0}$, the real part of the derivative of the eigenvalues with respect to the flow velocity $\mu_{\varepsilon}^{\prime}(0)$ is positive, and the first Lyapunov's coefficient $l_{1}(0)$ is negative. Therefore, the existence of flutter (i.e., the supercritical Hopf bifurcation) was demonstrated rigorously.

(iv) By comparing the bifurcation diagrams with different $l_{0}$, it is found that the flow velocities at which various bifurcations and chaos occur, increased with increasing the value of the nondimensional internal material length scale parameter $l_{0}$; that is, the internal material length scale parameter makes the microtube more stable.

\section{Data Availability}

The data used to support the findings of this study are available from the corresponding author upon request.

\section{Conflicts of Interest}

The author declares no conflicts of interest.

\section{Acknowledgments}

This work was supported by Guizhou Institute of Technology under Grant XJGC20190909.

\section{References}

[1] T. B. Benjamin, "Dynamics of a system of articulated pipes conveying fluid-I. Theory," Proceedings of the Royal Society of London. Series A. Mathematical and Physical Sciences, vol. 261, no. 1307, pp. 457-486, 1961.

[2] R. W. Gregory and M. P. Paidoussis, "Unstable oscillation of tubular cantilevers conveying fluid I. Theory," Proceedings of the Royal Society of London. Series A. Mathematical and Physical Sciences, vol. 293, no. 1435, pp. 512-527, 1966.

[3] M. P. Païdoussis and N. T. Issid, "Dynamic stability of pipes conveying fluid," Journal of Sound and Vibration, vol. 33, no. 3, pp. 267-294, 1974.

[4] P. J. Holmes, "Bifurcations to divergence and flutter in flowinduced oscillations: a finite dimensional analysis," Journal of Sound and Vibration, vol. 53, no. 4, pp. 471-503, 1977.

[5] J. Rousselet and G. Herrmann, "Dynamic behavior of continuous cantilevered pipes conveying fluid near critical velocities," Journal of Applied Mechanics, vol. 48, no. 4, pp. 943-947, 1981.

[6] N. S. Namchchivaya, "Non-linear dynamics of supported pipe conveying pulsating fluid-I. Subharmonic resonance," International Journal of Non-linear Mechanics, vol. 24, pp. 185196, 1989.

[7] N. S. Namchchivaya and W. M. Tien, "Non-linear dynamics of supported pipe conveying pulsating fluid-II. Combination 
resonance," International Journal of Non-linear Mechanics, vol. 24, no. 3, pp. 197-208, 1989.

[8] M. P. Païdoussis, G. X. Li, and F. C. Moon, "Chaotic oscillations of the autonomous system of a constrained pipe conveying fluid," Journal of Sound and Vibration, vol. 135, no. 1, pp. 1-19, 1989.

[9] L. Wang, "A further study on the non-linear dynamics of simply supported pipes conveying pulsating fluid," International Journal of Non-linear Mechanics, vol. 44, no. 1, pp. 115-121, 2009.

[10] P. J. Holmes, "Pipes supported at both ends cannot flutter," Journal of Applied Mechanics, vol. 45, no. 3, pp. 619-622, 1978.

[11] A. K. Bajaj, P. R. Sethna, and T. S. Lundgren, "Hopf bifurcation phenomena in tubes carrying a fluid," SIAM Journal on Applied Mathematics, vol. 39, no. 2, pp. 213-230, 1980.

[12] M. P. Paidoussis and C. Semler, "Nonlinear and chaotic oscillations of a constrained cantilevered pipe conveying fluid: a full nonlinear analysis," Nonlinear Dynamics, vol. 4, no. 6, pp. 655-670, 1994.

[13] J.-D. Jin, "Stability and chaotic motions of a restrained pipe conveying fluid," Journal of Sound and Vibration, vol. 208, no. 3, pp. 427-439, 1997.

[14] L. Wang and Q. Ni, "A note on the stability and chaotic motions of a restrained pipe conveying fluid," Journal of Sound and Vibration, vol. 296, no. 4-5, pp. 1079-1083, 2006.

[15] F. Liang and B. Wen, "Forced vibrations with internal resonance of a pipe conveying fluid under external periodic excitation," Acta Mechanica Solida Sinica, vol. 24, no. 6, pp. 477-483, 2011.

[16] B. Li, Z. Wang, and L. Jing, "Dynamic response of pipe conveying fluid with lateral moving supports," Shock and Vibration, vol. 2018, Article ID 3295787, 17 pages, 2018.

[17] Y.-L. Zhang, H.-R. Feng, and L.-Q. Chen, "Supercritical nonlinear vibration of a fluid-conveying pipe subjected to a strong external excitation," Shock and Vibration, vol. 2016, Article ID 3907498, 21 pages, 2016.

[18] S. Rinaldi, S. Prabhakar, S. Vengallatore, and M. P. Païdoussis, "Dynamics of microscale pipes containing internal fluid flow: damping, frequency shift, and stability," Journal of Sound and Vibration, vol. 329, no. 8, pp. 1081-1088, 2010.

[19] M. Najmzadeh, S. Haasl, and P. Enoksson, "A silicon straight tube fluid density sensor," Journal of Micromechanics and Microengineering, vol. 17, no. 8, pp. 1657-1663, 2007.

[20] D. Sparks, R. Smith, V. Cruz et al., "Dynamic and kinematic viscosity measurements with a resonating microtube," Sensors and Actuators A: Physical, vol. 149, no. 1, pp. 38-41, 2009.

[21] A. A. Bhirde, V. Patel, J. Gavard et al., "Targeted killing of cancer cells in vivo and in vitro with EGF-directed carbon nanotube-based drug delivery," ACS Nano, vol. 3, no. 2, pp. 307-316, 2009.

[22] L. Wang, "Size-dependent vibration characteristics of fluidconveying microtubes," Journal of Fluids and Structures, vol. 26, no. 4, pp. 675-684, 2010.

[23] T.-Z. Yang, S. Ji, X.-D. Yang, and B. Fang, "Microfluid-induced nonlinear free vibration of microtubes," International Journal of Engineering Science, vol. 76, pp. 47-55, 2014.

[24] M. Tang, Q. Ni, L. Wang, Y. Luo, and Y. Wang, "Nonlinear modeling and size-dependent vibration analysis of curved microtubes conveying fluid based on modified couple stress theory," International Journal of Engineering Science, vol. 84, pp. 1-10, 2014.

[25] S. K. Park and X.-L. Gao, "Bernoulli-Euler beam model based on a modified couple stress theory," Journal of
Micromechanics and Microengineering, vol. 16, no. 11, pp. 2355-2359, 2006.

[26] W. Xia and L. Wang, "Microfluid-induced vibration and stability of structures modeled as microscale pipes conveying fluid based on non-classical Timoshenko beam theory," Microfluidics and Nanofluidics, vol. 9, no. 4-5, pp. 955-962, 2010.

[27] M. Asghari, M. H. Kahrobaiyan, and M. T. Ahmadian, “A nonlinear Timoshenko beam formulation based on the modified couple stress theory," International Journal of Engineering Science, vol. 48, no. 12, pp. 1749-1761, 2010.

[28] N. A. Fleck, G. M. Muller, M. F. Ashby, and J. W. Hutchinson, "Strain gradient plasticity: theory and experiment," Acta Metallurgica et Materialia, vol. 42, no. 2, pp. 475-487, 1994.

[29] D. C. C. Lam, F. Yang, A. C. M. Chong, J. Wang, and P. Tong, "Experiments and theory in strain gradient elasticity," Journal of the Mechanics and Physics of Solids, vol. 51, no. 8, pp. 1477-1508, 2003.

[30] A. W. McFarland and J. S. Colton, "Role of material microstructure in plate stiffness with relevance to microcantilever sensors," Journal of Micromechanics and Microengineering, vol. 15, no. 5, pp. 1060-1067, 2005.

[31] F. Yang, A. C. M. Chong, D. C. C. Lam, and P. Tong, "Couple stress based strain gradient theory for elasticity," International Journal of Solids and Structures, vol. 39, no. 10, pp. 2731-2743, 2002.

[32] D. Y. Cao and Y. Q. Wang, "Wave dispersion in viscoelastic lipid nanotubes conveying viscous protein solution," The European Physical Journal Plus, vol. 135, no. 1, p. 24, 2020.

[33] Y. Q. Wang, H. H. Li, Y. F. Zhang, and J. W. Zu, “A nonlinear surface-stress-dependent model for vibration analysis of cylindrical nanoscale shells conveying fluid," Applied Mathematical Modelling, vol. 64, pp. 55-70, 2018.

[34] Z. H. Li and Y. Q. Wang, "Vibration and stability analysis of lipid nanotubes conveying fluid," Microfluidics and Nanofluidics, vol. 23, no. 11, p. 12, 2019.

[35] M. Hosseini and R. Bahaadini, "Size dependent stability analysis of cantilever micro-pipes conveying fluid based on modified strain gradient theory," International Journal of Engineering Science, vol. 101, pp. 1-13, 2016.

[36] R. Bahaadini and M. Hosseini, "Effects of nonlocal elasticity and slip condition on vibration and stability analysis of viscoelastic cantilever carbon nanotubes conveying fluid," Computational Materials Science, vol. 114, pp. 151-159, 2016.

[37] K. Hu, Y. K. Wang, H. L. Dai, L. Wang, and Q. Qian, "Nonlinear and chaotic vibrations of cantilevered micropipes conveying fluid based on modified couple stress theory," International Journal of Engineering Science, vol. 105, pp. 93-107, 2016.

[38] Y. Guo, J. Xie, and L. Wang, “Three-dimensional vibration of cantilevered fluid-conveying micropipes-types of periodic motions and small-scale effect," International Journal of Nonlinear Mechanics, vol. 102, pp. 112-135, 2018.

[39] C. Semler, G. X. Li, and M. P. Päidoussis, "The non-linear equations of motion of pipes conveying fluid," Journal of Sound and Vibration, vol. 169, no. 5, pp. 577-599, 1994.

[40] S. Kong, S. Zhou, Z. Nie, and K. Wang, "The size-dependent natural frequency of Bernoulli-Euler micro-beams," International Journal of Engineering Science, vol. 46, no. 5, pp. 427-437, 2008.

[41] G. X. Li and M. P. Paidoussis, "Stability, double degeneracy and chaos in cantilevered pipes conveying fluid," International Journal of Non-linear Mechanics, vol. 29, no. 1, pp. 83-107, 1994. 
[42] Y. A. Kuznetsov, Elements of Applied Bifurcation Theory, Springer-Verlag, Berlin, Germany, Second edition, 1998.

[43] G. Iooss and D. D. Joseph, Elementary Stability and Bifurcation Theory, Springer-Verlag, Berlin, Germany, Second edition, 1990.

[44] Y. Yue and J.-H. Xie, "Symmetry, cusp bifurcation and chaos of an impact oscillator between two rigid sides," Applied Mathematics and Mechanics, vol. 28, no. 8, pp. 1109-1117, 2007. 